\title{
Simulation of transonic flows through a turbine blade cascade with various prescription of outlet boundary conditions
}

\author{
Petr Louda ${ }^{1, \mathrm{a}}$, Petr Straka $^{2}$, Jaromír Př́hoda ${ }^{3}$ \\ ${ }^{1, a}$ Faculty of Mechanical Engineering, Czech Technical University in Prague, Czech Republic \\ ${ }^{2}$ VZLU Czech Aerospace Research Centre, Plc, Prague, Czech Republic \\ ${ }^{2}$ Institute of Thermomechanics AS CR, v.v.i., Prague, Czech Republic
}

\begin{abstract}
The contribution deals with the numerical simulation of transonic flows through a linear turbine blade cascade. Numerical simulations were carried partly for the standard computational domain with various outlet boundary conditions by the algebraic transition model of Straka and Príhoda [1] connected with the EARSM turbulence model of Hellsten [2] and partly for the computational domain corresponding to the geometrical arrangement in the wind tunnel by the $\gamma-\zeta$ transition model of Dick et al. [3] with the SST turbulence model. Numerical results were compared with experimental data. The agreement of numerical results with experimental results is acceptable through a complicated experimental configuration.
\end{abstract}

\section{Introduction}

The problem of the shock-wave/boundary-layer interaction is studied in the long term nevertheless the majority of results are focused on the interaction with the turbulent boundary layer. A review of results related to the shock-wave/boundary layer interaction is given e.g. by Gaitonde [4] and/or Arnal and Délery [5].

A relatively significant attention is recently given to the problem of the separation-induced transition due to the interaction of the shock wave with the laminar boundary layer, as e.g. experimental and numerical investigations of the interaction in the framework of the TFAST project "Transition Location Effect on Shock Wave Boundary Layer Interaction“, see Flaszynski et al. [6] and Piotrowicz et al. [7].

The structure of the transonic flow through a turbine blade cascade considerably depends on the character of the shock-wave/boundary-layer interaction. In case of the laminar boundary layer, the shock-wave/boundary-layer interaction is usually connected with the flow separation followed by the transition in separated flow.

Existing simulations of transonic flows through a linear turbine blade cascade have shown a rather significant dependence of results on the prescription of the outlet boundary condition, i.e. outlet static pressure. Due to the relatively complicated geometrical arrangement of the test section with the immediately connecting settling chamber, the outlet of the computational domain is usually prescribed in the traversing plane behind the blade cascade where the static pressure is given on the basis of mean values of measured data.

Therefore there were realized numerical simulations partly for the computational domain corresponding to the geometrical arrangement of the wind tunnel by the $\gamma-\zeta$

\footnotetext{
$\overline{{ }^{a} \text { Corresponding author: petr.louda } @ f s . c v u t . c z}$
}

transition model of Dick et al. [3] connected with the SST turbulence model and partly for the standard computational domain with various outlet boundary conditions by the algebraic transition model of Straka and Príhoda [1] connected with the EARSM turbulence model. Numerical simulations were carried out for transonic flow through a linear turbine blade cascade corresponding to a rotor mid-section of the last stage of a large output steam turbine.

\section{Mathematical model}

The numerical simulation of compressible flows is based on the solution of the conditionally-averaged NavierStokes equations completed by the constitutional relations and by a turbulence model, a model of turbulent heat transfer and by a model of the laminar/turbulent transition. The basic mathematical model consists of the EARSM turbulence model of Hellsten [2], the algebraic transition model proposed by Straka and Př́íhoda [1] and the turbulent heat transfer model based on the generalized gradient hypothesis according to Launder [8].

The EARSM turbulence model was used in the form corresponding to models with the turbulent viscosity. Turbulent scales are expressed by the turbulent energy $k$ and by the specific dissipation rate $\omega$ by means of the SST turbulence model of Menter [9]. Turbulent time scale $\tau \sim 1 / \omega$ near the wall is given by the Kolmogorov viscous time scale. The production term in the turbulent energy equation is modified for the reduction of the undesirable overproduction of the turbulent energy in the stagnation region. Production and destruction terms in the equation for the turbulent energy are multiplied by the intermittency coefficient $\gamma$. Similarly, the effective viscosity is given by $\mu_{e f}=\mu+\gamma \mu_{t}$ in the transition region. 
The transition model is based on the concept of different values of the intermittency coefficient in the boundary layer $\gamma_{i}$ and in the free stream $\gamma_{e}$. The intermittency coefficient in the boundary layer $\gamma_{i}$ is described by the relation

$$
\gamma_{i}=1-\exp \left[-\hat{n} \sigma\left(R e_{x}-R e_{x t}\right)^{2}\right]
$$

according to Narasimha [10]. The transition onset is given by the empirical correlation for the momentum Reynolds number $R e_{\theta t}=\mathrm{f}\left(T u, \lambda_{t}\right)$ where $T u(\%)$ is the free-stream turbulence level and $\lambda_{t}$ is the pressuregradient parameter. The transition length is expressed using the parameter $N=\hat{n} \sigma R e_{\theta t}{ }^{3}$ where $\hat{n}$ is the spot generation rate and $\sigma$ is the spot propagation rate introduced by Narasimha [10]. The parameter $N$ for the attached flow is similarly given by the empirical relation $N=\mathrm{g}\left(T u, \lambda_{t}\right)$ proposed by Solomon et al. [11]. The onset of transition in separated flow is given by the correlation according to Mayle [12] in the form

$$
R e_{x t}=300 R e_{\theta s}^{0.7}+R e_{x s}
$$

where $R e_{\theta s}$ is the momentum Reynolds number at the separation and $R e_{x s}$ is the Reynolds number related to the distance of the separation from the leading edge. The transition length of the separation is given according to Walker [13] by the relation

$$
\hat{n} \sigma=\frac{R e_{x t}^{-1.34}}{40}
$$

and so the same approach can be applied as in the attached flow.

For prediction of transitional flows in complex geometries, the application of local variables is necessary. The momentum Reynolds number is replaced according to Langtry and Menter [14] by the maximum of the vorticity Reynolds number in the form

$$
\operatorname{Re}_{\Omega}=y^{2}|\Omega| / v
$$

where $y$ is the distance from the wall and $\Omega$ is the absolute value of the vorticity tensor. The link between both Reynolds numbers is expressed by the relation $\operatorname{Re}_{\theta}=$ $R e_{\Omega \max } / C$ where the parameter $C$ depends on the pressure gradient. The variation of the parameter $C$ with the pressure gradient parameter was estimated by means of similar solutions of Falkner-Skan velocity profiles.

The mathematical model was implemented into the in-house numerical code based on the finite volume method. The multi-block quadrilateral structured grid with a block overlapping implementation refined near walls was applied for numerical simulations.

The $\gamma-\zeta$ transition model according to Dick et al. [3] (see also Lodefier and Dick [15]) is based on two transport equations for the near-wall part $\gamma$ and freestream part of the intermittency $\zeta$ used in the form

$$
\begin{aligned}
& \frac{\partial(\rho \gamma)}{\partial t}+\frac{\partial\left(\rho U_{i} \gamma\right)}{\partial x_{i}}=P_{\gamma}+\frac{\partial}{\partial x_{i}}\left[\left(\mu+\sigma_{\gamma} \mu_{t}\right) \frac{\partial \gamma}{\partial x_{i}}\right] \\
& \frac{\partial(\rho \zeta)}{\partial t}+\frac{\partial\left(\rho U_{i} \zeta\right)}{\partial x_{i}}=-E_{\zeta}+\frac{\partial}{\partial x_{i}}\left[\left(\mu+\sigma_{\zeta} \mu_{t}\right) \frac{\partial \zeta}{\partial x_{i}}\right]
\end{aligned}
$$

with the production term in the eq. (5)

$$
P_{\gamma}=2 \beta_{\gamma}(1-\gamma) \sqrt{-\ln (1-\gamma)} \rho U_{\gamma} F_{s}
$$

where $F_{s}$ is a starting function set to unity at the start of transition. The dissipation term in the eq. (6) is given by the relation

$$
E_{\zeta}=C_{2} \mu_{\zeta} \frac{U}{U_{e}^{2}} \frac{\partial U}{\partial n} \frac{\partial \zeta}{\partial n}
$$

where $U$ is the local velocity and $U_{e}$ is the mean velocity at the boundary layer edge. The term $E_{\zeta}$ guarantees that $\zeta$ vanishes in the boundary layer, so that both intermittencies are complementary in the turbulent flow. The relation between the growth parameter $\beta_{\gamma}$ and the spot growth rate is

$$
\beta_{\gamma}=\sqrt{\frac{N \sigma}{U_{e}}}
$$

The boundary conditions are $\gamma=0, \zeta=1$ in the inlet, $\partial \zeta / \partial n=\partial \gamma / \partial n=0$ in the outlet and $\partial \gamma / \partial n=0, \zeta=0$ on the wall. The criteria of Abu-Ghannam and Shaw [16] and Mayle [12] are used for the transition onset in the attached flow. For transition in separated flow the Mayle criterion modified by Dick et al. [3] was used in the form

$$
R e_{s t}=700 R e_{\theta s}^{0.7} \exp \left(-0.3 T u_{e}\right)
$$

with parameters related to the separation point. The used criteria are functions of boundary layer parameters as the momentum thickness, free-stream velocity and turbulence intensity. The used transition model and its implementtation into the in-house numerical code are described in detail by Louda et al. [17].

\section{Results}

The both transition models i.e. the EARSM turbulence model connected with the algebraic transition model and the SST turbulence model with the $\gamma-\zeta$ transition model were applied for the numerical simulation of $2 \mathrm{D}$ transonic flow through a linear turbine blade cascade corresponding to a rotor mid-section of the last stage of a large output steam turbine. Numerical results were compared with experimental results covering optical and pressure measurements, see Luxa et al. [18].

The predictions were carried out for the relative spacing $t / c=0.718$, inlet angle $\alpha_{1}=29.1^{\circ}$ and for various outlet isentropic Mach number $M_{2 i s}$ corresponding to the static pressure $p_{2}$ determined by the data reduction method in the traversing plane behind the blade cascade.

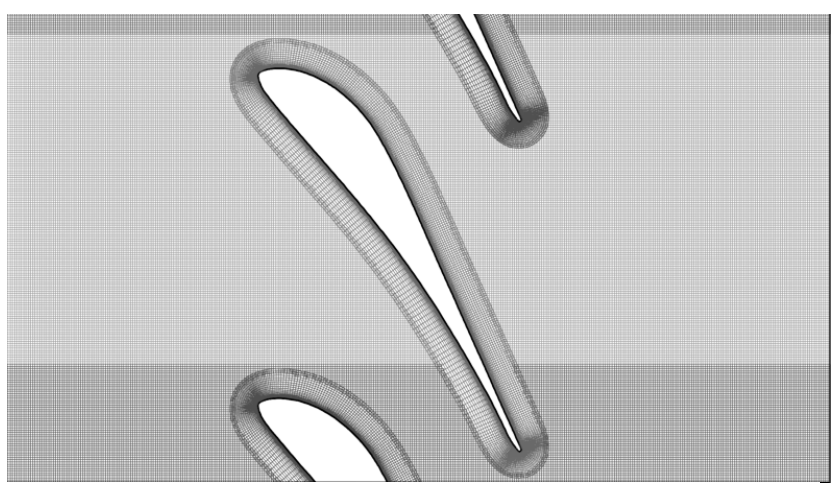

Fig. 1. Scheme of the computational domain 
The multi-block quadrilateral structured computational mesh refined near walls was used for numerical simulations by means the algebraic transition model implemented into the in-house numerical code. The computational domain is demonstrated in Fig. 1. This approach allows the application of structured grids for complex geometries as well.

The inlet of the domain was in the distance of $0.55 c$ from the blade leading edge and the outlet in the distance $0.7 c$ behind trailing edges. The inlet boundary conditions were prescribed by the constant total pressure, total temperature and inlet flow angle. The outlet boundary condition was defined by the constant static pressure determined according to the outlet isentropic Mach number. Periodicity conditions were applied on corresponding free side boundaries of the computation domain. The inlet free-stream turbulence parameters corresponding to turbulence intensity $T u=1.5 \%$ and the ratio of the turbulent and molecular viscosity $\mu_{t} / \mu=50$ were chosen.

Predictions were focused on the adequate simulation of the shock-wave/boundary-layer interaction. The flow separation on the blade suction side is caused by the interaction of the reflected shock wave with the laminar boundary layer.

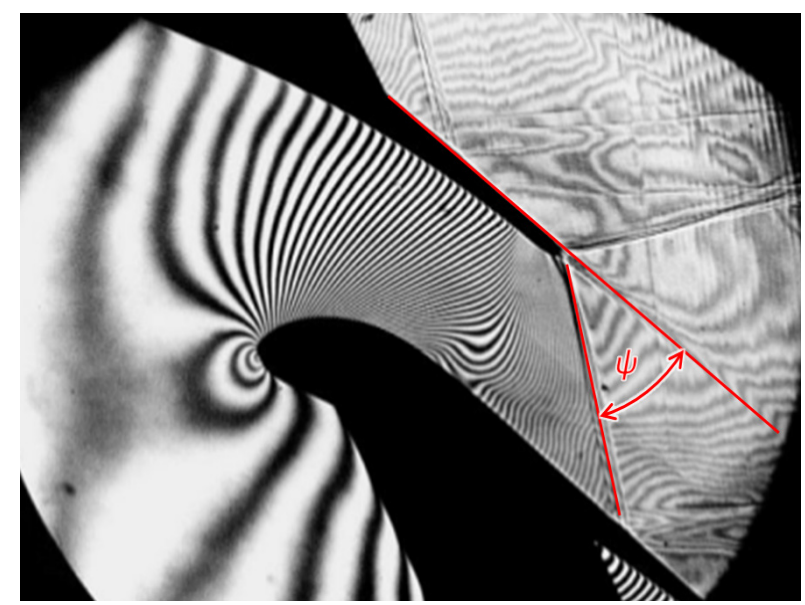

Fig. 2. Interferometric picture for $M_{2 i s}=1.515$

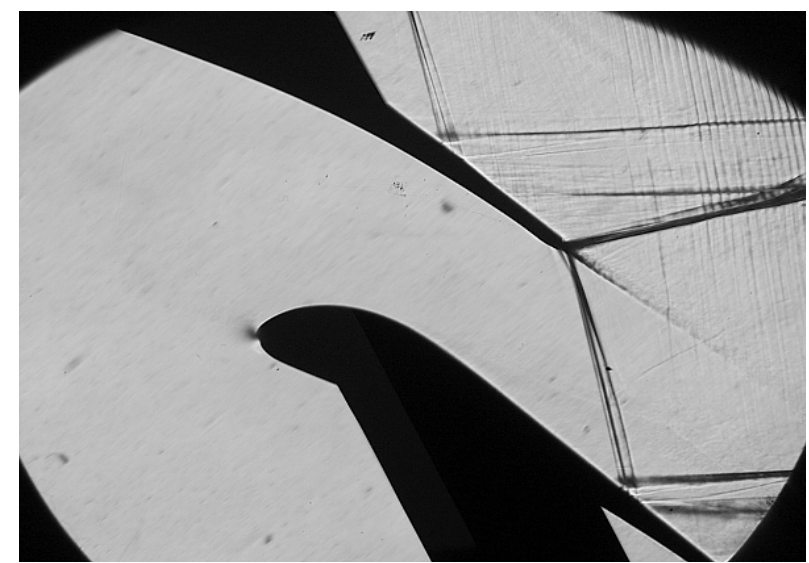

Fig. 3. Schlieren picture for $\mathrm{M}_{2 \mathrm{is}}=1.515$

The interferometric and schlieren pictures obtained for $M_{2 i s}=1.515$ are shown in Fig. 2 and 3. The interaction of the inner branch of the exit shock wave with the boundary layer on the suction side is connected with the separation on the laminar boundary layer.
Simulations by means of the algebraic transition model were carried out for various outlet static pressures $p_{2} / p_{01}$ i.e. for various Mach numbers $M_{2 i s}$ with the aim to reach a good agreement of the predicted flow field in the blade cascade expressed by the Mach number isolines. This agreement was tested by the position of the inner branch of the reflected shock wave especially by the angle $\psi$ between the shock wave and the linear part of the blade suction side, see Fig. 2.

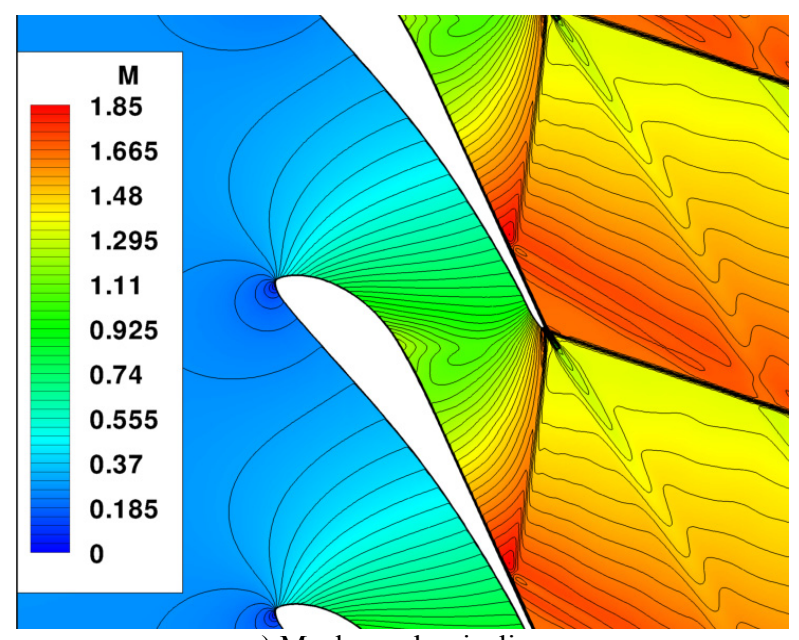

a) Mach number isolines

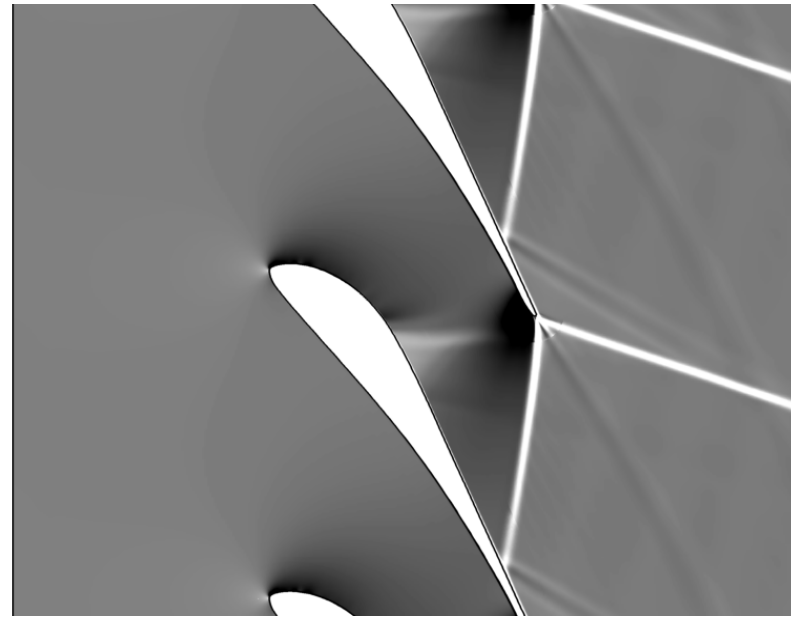

b) Numerical schlieren

Fig. 4. Flow field for $M_{2 i s}=1.515$ (algebraic transition model)

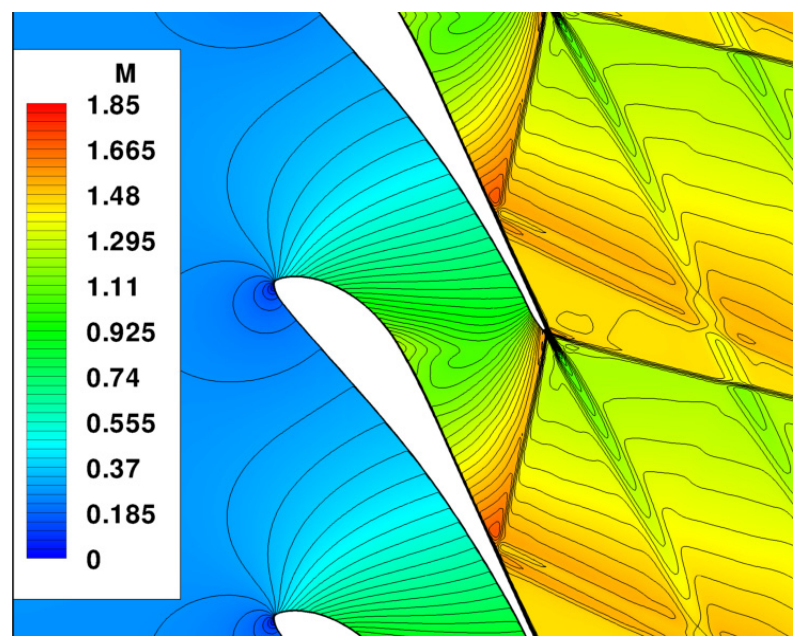

a) Mach number isolines 


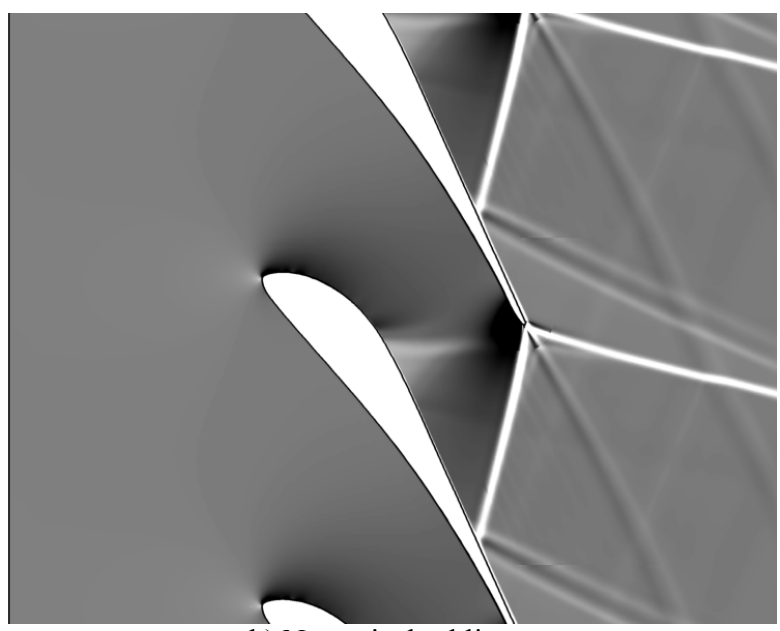

b) Numerical schlieren

Fig. 5. Flow field for $M_{2 i s}=1.422$ (algebraic transition model)

The best agreement was achieved for the pressure ratio $p_{2} / p_{01}=0.3048$ corresponding to $M_{2 i s}=1.422$. The Mach number isolines and numerical schlieren pictures obtained by the algebraic transition model are shown for $M_{2 i s}=1.422$ and 1.515 in Fig. 4 and 5. The variation of the shock wave angle with the pressure ratio $p_{2} / p_{01}$ is presented in Fig. 6.

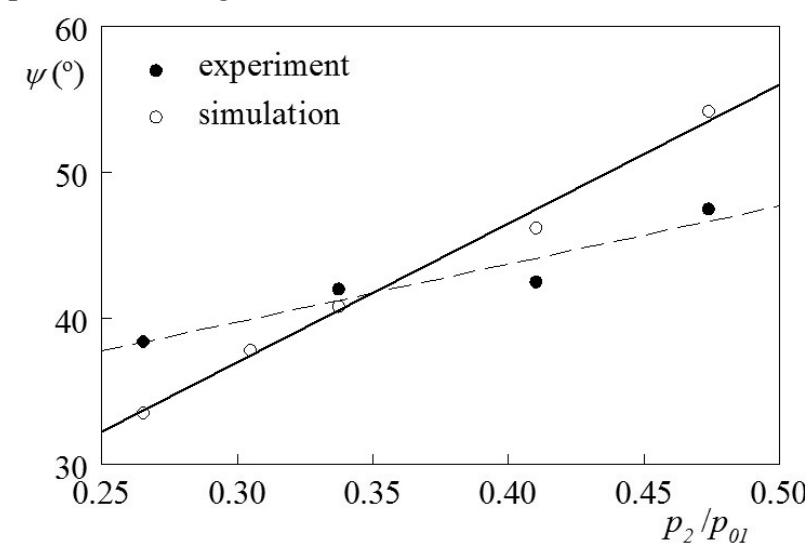

Fig. 6. Variation of the shock wave angle with the outlet static pressure

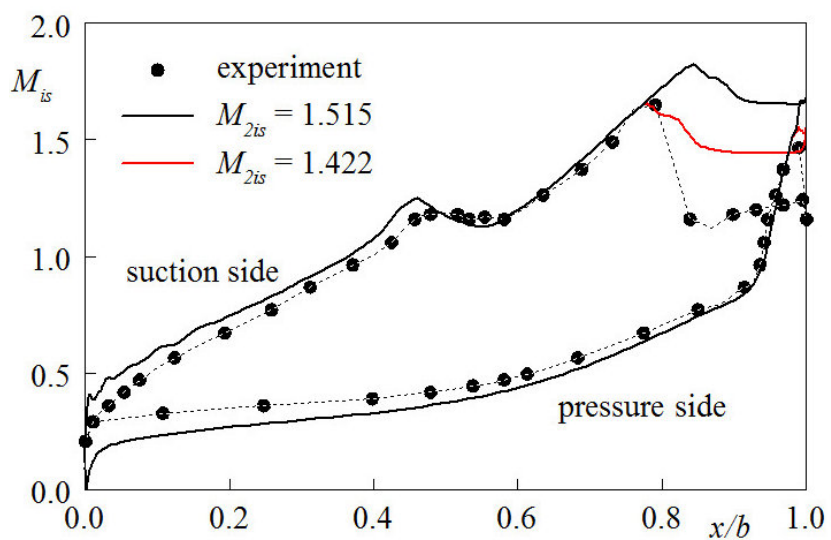

Fig. 7. Distribution of the isentropic Mach number along the blade (algebraic transition model)

The distribution of the isentropic Mach number along the blade surface is compared with experimental data in Fig. 7. The distance $b$ represents the axial chord length. The only difference between the both Mach numbers $M_{2 i s}$ is the position of the shock-wave impact on the suction side where the prediction for $M_{2 i s}=1.422$ agrees well with experiments.

The distribution of the skin friction on the blade surface is presented in Fig. 8. The skin friction coefficient is defined by the relation $C_{f}=\tau_{w} /\left(p_{01}-p_{1}\right)$. The skin friction distribution reflects the Mach number distribution as the only difference is downstream of the predicted shock-wave impact.

The detail of skin friction distribution with separation zones is given in Fig. 9. The separation zone estimated from experimental results for the $M_{2 i s}=1.515$ is marked by the rectangle. The extent of the separation zone is approximately from $x / b \approx 0.771$ to 0.832 . The interaction of the inner branch of the exit shock wave with the boundary layer on the suction side and the separation zone is acceptably predicted even though the predicted separation length is rather shorter.

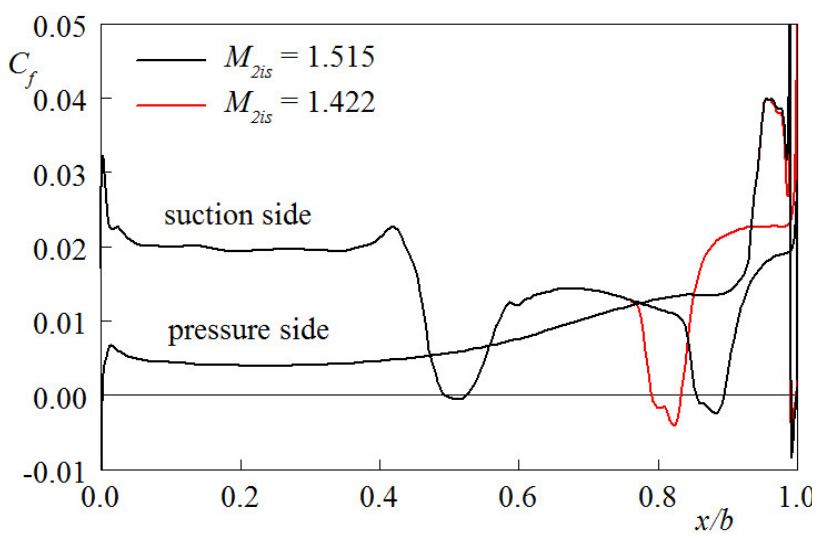

Fig. 8. Skin friction distribution (algebraic transition model)

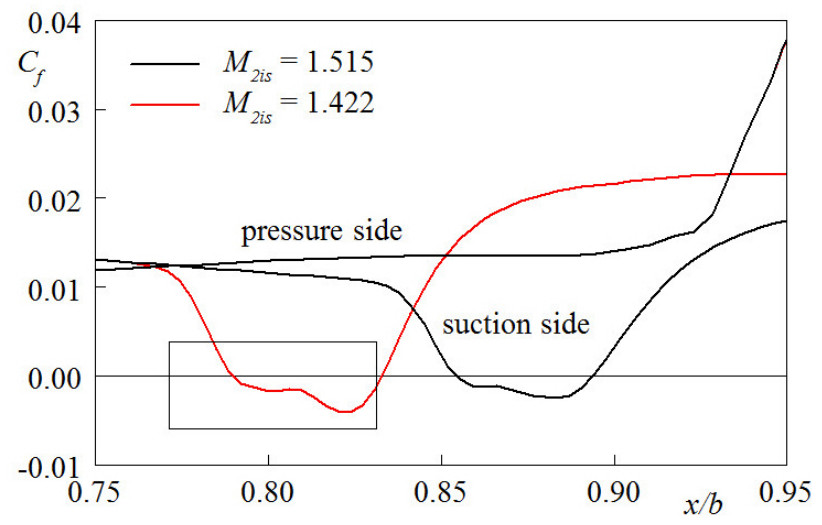

Fig. 9. Detail of the skin friction distribution (algebraic transition model)

Further, it can be seen from the distribution of the Mach number and the skin friction that the sudden change of the surface curvature of the suction side at the distance $x / b \approx 0.55$ leads to a short laminar separation bubble. Besides, a very short separation can be seen as well on the blade pressure side just upstream of the trailing edge, see Fig. 8.

The computational domain used for application of the $\gamma-\zeta$ transition model covers the inlet part, the test section with six blades and a part of the settling chamber. The upper blade is extended by the perforated tailbox. The chosen computational domain corresponds as much as possible to the experimental arrangement in the wind tunnel. 


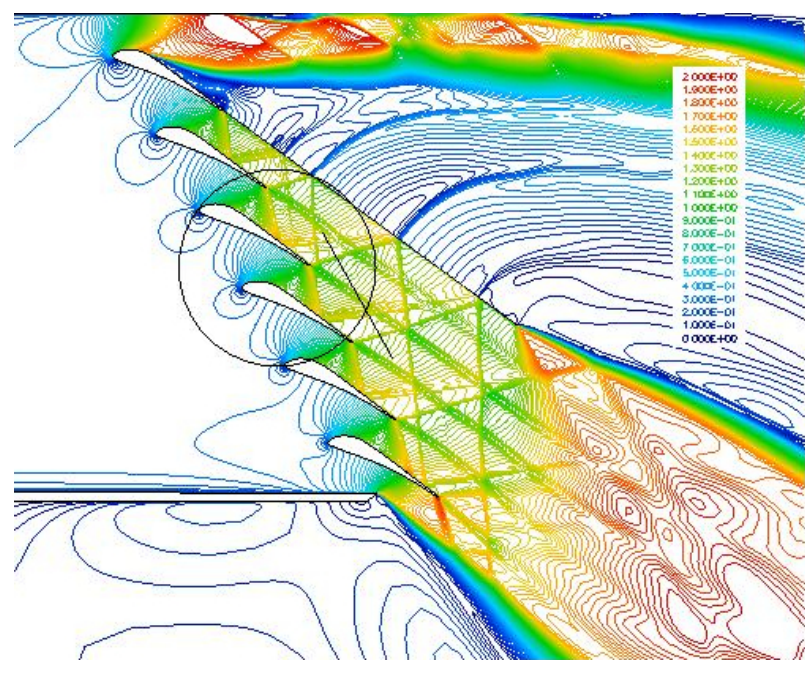

Fig. 10. Mach number isolines $(\gamma-\zeta$ transition model $)$

A cell-centered finite volume method with quadrilateral (in 2D) or hexahedral (in 3D) finite volumes composing a structured grid was used for the spatial discretization of the $\gamma-\zeta$ transition model. The inlet boundary conditions were prescribed in the same way as for the algebraic transition model. Due to the used configuration, the prescription of the outlet boundary condition is somewhat difficult and so the trial-and-error method was used.

The results obtained for the ratio $p_{2} / p_{01}=0.300$ corresponds well with experimental data for the $M_{2 i s}=$ 1.356 related to the static pressure in the traversing plane. The whole configuration is apparent from Fig. 10 where the Mach number isolines are shown. The circle marks the optical window in the test section and the line is the traversing plane.

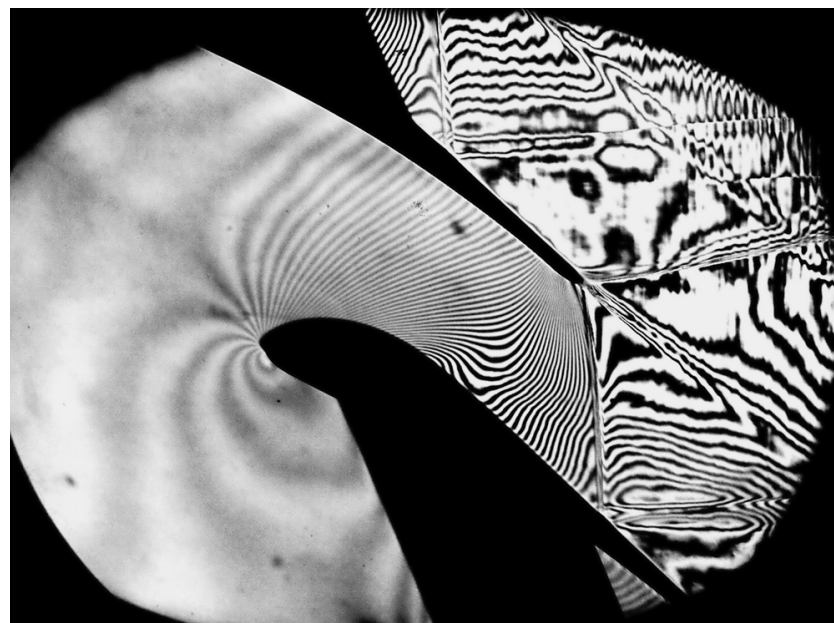

Fig. 11. Interferometric picture for $M_{2 i s}=1.356$

The interferometric picture for the isentropic outlet Mach number $M_{2 i s}=1.356$ is shown in Fig. 11. The detail of the Mach number isolines corresponding to the measured area is shown in Fig. 12. The agreement of the both flow fields is acceptable. The distribution of the isentropic Mach number along the blade is presented for the two middle blades in Fig. 13. It can be seen that the interaction of the inner branch of the outlet shock wave with the boundary layer is on the both blades somewhat different including the shock wave angle.

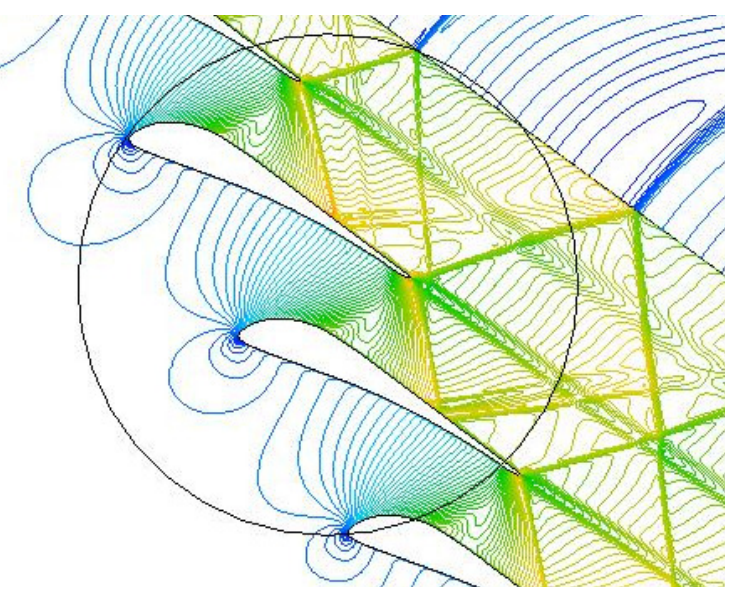

Fig. 12. Detail of the Mach number isolines $(\gamma-\zeta$ transition model)

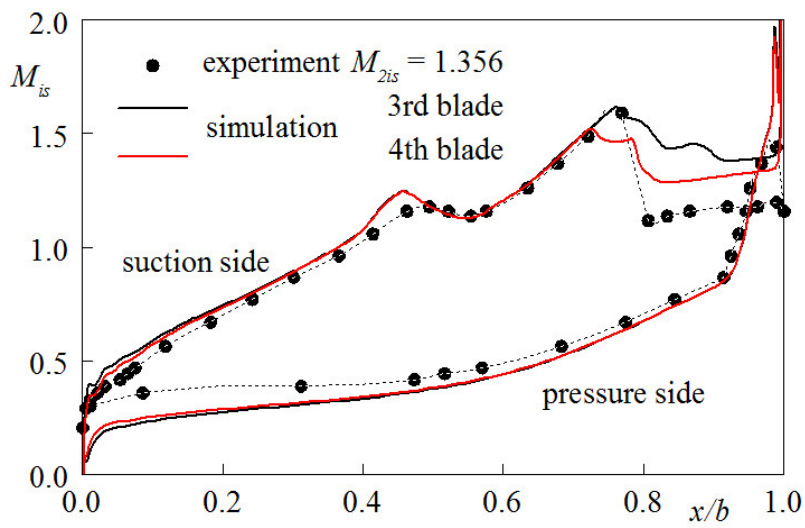

Fig. 13. Distribution of the isentropic Mach number $(\gamma-\zeta$ transition model)

The corresponding distribution of the skin friction coefficient is shown in Fig. 14 where the separation position agrees with the shock wave impact. The detail of the skin friction distribution is shown in Fig. 15. The experimentally determined separation zone covering the suction side form from $x / b \approx 0.75$ to 0.817 is marked by the rectangle.

The agreement of the prediction of the separation location with experimental data is quite acceptable. Similarly as the algebraic transition model, the $\gamma-\zeta$ transition model predicts a nearly separation in the region of the sudden change of the surface curvature of the suction side and a very short separation near the trailing edge on the blade pressure side.

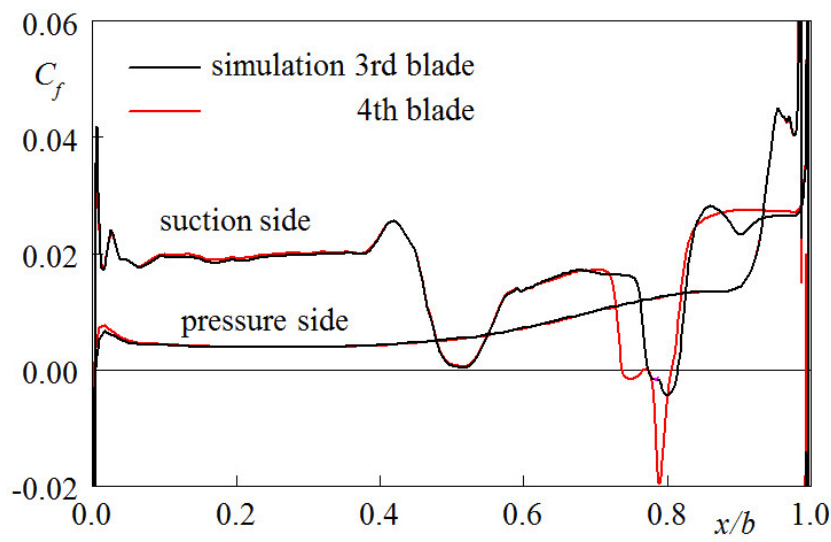

Fig. 14 Skin friction distribution $(\gamma-\zeta$ transition model $)$ 


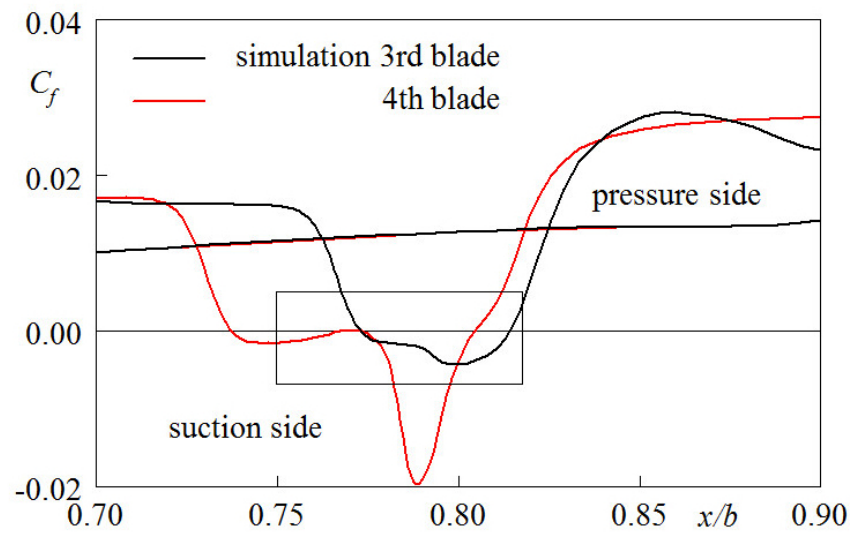

Fig. 15 Detail of the skin friction distribution $(\gamma-\zeta$ transition model)

\section{Conclusions}

Numerical simulations of 2D transonic flow through a linear mid-span turbine blade cascade were carried out for various outlet boundary conditions. Predictions were accomplished partly for the standard computational domain with various outlet boundary conditions by the algebraic transition model of Straka and Př́ihoda [1] connected with the EARSM turbulence model of Hellsten [2] and partly for the computational domain corresponding to the geometrical arrangement in the wind tunnel by the $\gamma-\zeta$ transition model of Dick et al. [3] with the SST turbulence model.

The accomplished simulations have shown the significant dependence of results on the prescription of the outlet boundary condition. Simulations carried out for the standard computational domain with the output at the traversing plane behind the blade cascade and/or for the computational domain corresponding to the experimental arrangement have shown that numerical results obtained on the basis of the numerical optimization correspond quite well to experimental data. The agreement of predicted flow fields and main parameters including energy losses is acceptable.

The work was supported by the Technology Agency of the Czech Republic under the grants TH02020057 and TA04020129. Institutional support RVO 61388998 is also gratefully acknowledged.

\section{References}

1. P. Straka, J. Př́íhoda, Proc. Conf. Topical Problems of Fluid Mechanics, Prague, 131-134 (2014)

2. A. Hellsten, New two-equation turbulence model for aerodynamics applications, $\mathrm{PhD}$. Thesis, Helsinki University of Technology (2004)

3. E. Dick, S. Kubacki, K. Lodefier, W. Elsner, Intermittency modelling of transitional boundary layer flows on steam and gas turbine blades, Engineering Applications of Computational Fluid Dynamics, Vol. 2, International Energy and Environment Foundation. 173-216 (2012)
4. D. V. Gaitonde, Progress in Aerospace Sciences, 72, 80-99 (2015)

5. D. Arnal, J. Délery, Laminar-turbulent transition and shock wave/boundary layer interaction, RTOEN-AVT-116, 46 p. (2005)

6. P. Flaszynski, P. Doerffer, R. Szwaba, P. Kaczynski, M. Piotrowicz, Jour. of Thermal Science, 24, 510515 (2015)

7. M. Piotrowicz, P. Flaszyński, Jour. of Physics: Conference Series, 760, 012023 (2016)

8. B. E. Launder, Jour. of Heat Transfer, 110, 11121128, (1988)

9. F. R. Menter, AIAA Jour., 32, 1598-1605 (1994)

10. R. Narasimha, Prog. Aerospace Science, 22, 29-80, (1985)

11. W. J. Solomon, G. J. Walker, J. P. Gostelow, Jour. of Turbomachinery, 118, 744-751 (1996)

12. R. E. Mayle, Jour. of Turbomachinery, 113, 509-537 (1991)

13. G. J. Walker, AIAA Jour., 27, 595-607 (1989)

14. R. Langtry, F. R. Menter, AIAA Jour., 47, 28942906 (2009)

15. K. Lodefier, E. Dick, Flow, Turbulence and Combustion, 76, 103-132 (2006)

16. B. J. Abu-Ghannam, R. Shaw, Jour. of Mech. Eng. Science, 22, 213-228 (1980)

17. P. Louda, J. Př́íhoda, K. Kozel, Proc. Conf. Topical Problems of Fluid Mechanics, Prague, 211-220 (2017)

18. M. Luxa, D. Šimurda, P. Šafařík, J. Synáč, B. Rudas, Proc. 10th European Conference on Turbomachinery, Fluid Dynamics and Thermo-Dynamics, Lappeenranta, 360-369 (2013) 